\title{
Formation of synovial joints and articular cartilage
}

\author{
S. Moskalewski, A. Hyc, E. Jankowska-Steifer, A. Osiecka-Iwan \\ Department of Histology and Embryology, Warsaw Medical University, Warsaw, Poland
}

[Received 1 March 2013; Accepted 29 March 2013]

Chondrocytes differentiate from mesenchymal progenitors and produce templates (anlagen) for the developing bones. Chondrocyte differentiation is controlled by Sox transcription factors. Templates for the neighbour bones are subsequently separated by conversion of differentiated chondrocytes into non-chondrogenic cells and emergence of interzone in which joints cavitation occurs. A central role in initiating synovial joint formation plays Wnt-14/beta-catenin signalling pathway. Moreover, bone morphogenetic proteins and growth and differentiation factors are expressed at the site of joint formation. Joint cavitation is associated with increased hyaluronic acid synthesis. Hyaluronic acid facilitates tissue separation and creation of a functional joint cavity. According to the traditional view articular cartilage represents part of cartilage anlage that is not replaced by bone through endochondral ossification. Recent studies indicate, however, that peri-joint mesenchymal cells take part in interzone formation and that these interzone cells subsequently differentiate into articular chondrocytes and synovial cells. Thus, anlage chondrocytes have a transient character and disappear after cessation of growth plate function while articular chondrocytes have stable and permanent phenotype and function throughout life. (Folia Morphol 2013; 72, 3: 181-187)

Key words: cartilage anlage, joint interzone, joint cavitation, peri-joint mesenchymal cells

\section{FORMATION OF CARTILAGINOUS TEMPLATES}

The long bones of the vertebrate appendicular skeleton arise from initially continuous condensations of mesenchymal cells that subsequently differentiate, segment and cavitate to form discrete elements separated by synovial joint (Fig. 1) [23]. From mesenchymal progenitors differentiate chondrocytes which produce the templates, or cartilage anlagen, for the developing bones [19]. Development of embryonic cartilage primordia and growth plates occur under control of SOX-5, SOX-6 and SOX-9 transcription factors [1, 37]. The SOX gene family encodes a group of transcription factors defined by the conserved high mobility group DNA-binding domain. SOX-9 is absolutely required for cell survival in precartilaginous condensations and for chondrocyte differentiation in cartilage primordia $[1,7]$. SOX-5 and SOX-6 are not absolutely required for chondrocyte differentiation, but strongly potentiate SOX-9's chondrogenic activity [22] and in synovial joint morphogenesis promote both growth plate and articular chondrocytes differentiation [12].

\section{EMERGENCE OF INTERZONE}

Cartilage templates of neighbour bones are separated through development of non-cartilaginous region known as the interzone. The interzone is essential for joint formation since its removal by microdissection led to joint ablation and fusion of bones [25]. The segmentation process that generates 


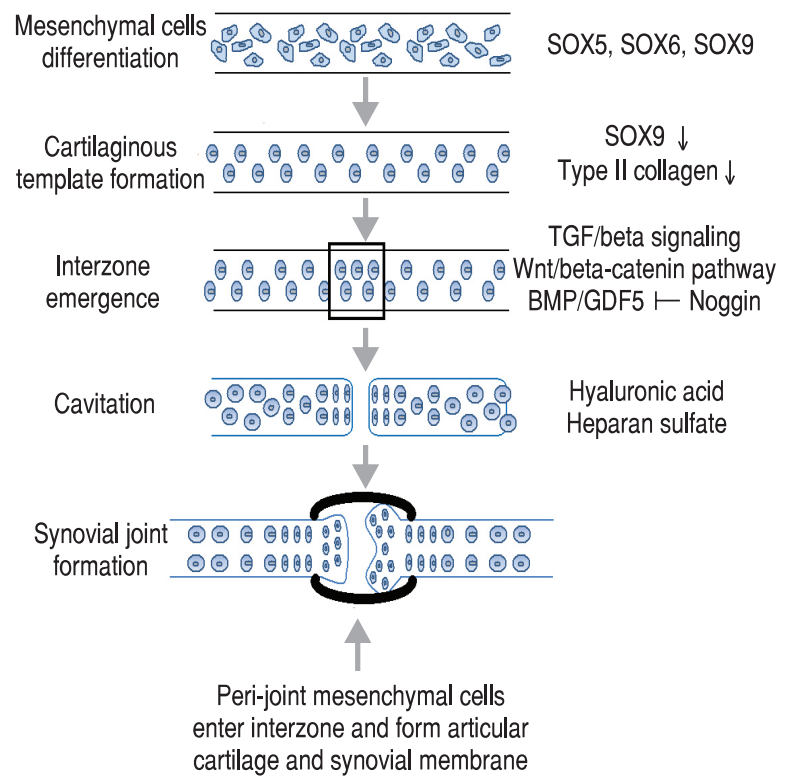

Figure 1. The main steps in synovial join formation.

joints between the skeletal elements is initiated by the conversion of differentiated chondrocytes at the sites of presumptive joint formation into the densely packed cells of the joint interzone. The first step in joint development is the specification of articular progenitor cells $[45,52]$. In contrary to determination, specification is a reversible process associated with a capability of cells to differentiate autonomously in neutral environment, but not in non-neutral environment. It occurs in the embryo once skeletogenic mesenchymal cells have condensed into precartilaginous masses. Most cells in these masses commit to the growth plate chondrocyte fate, while specific subpopulations commit to the articular fate and go on to develop into one or another synovial joint cell type [12]. During interzone formation articular progenitor cell specification prompts the downregulation of the gene for the master chondrogenic transcription factor SOX-9 [29]. It is accompanied by downregulation in expression of type II collagen and other cartilage-specific genes $[3,8,17]$. The repressive signals that prevent chondrogenic differentiation within interzone are likely to promote differentiation along a fibrous lineage. It may continue until other components like menisci or ligaments in knee joint are produced and the joint forms a "mini organ" of differentiated tissues [3]. Once formed, interzone differentiate in 3 layers: 2 chondrogenic, perichondrium-like layers that cover the cartilaginous surfaces and 1 intermediate layer of loose, avascular mesenchyme that separates them and continues peripherally in a vascular mesenchyme, the so-called synovial mesenchyme $[2,42]$. Within interzone, along planes destined to become the articular surfaces of synovial joints cavitation occurs [14].

Several signalling pathways work in a cascade to specify the fate of joint forming cells. A major signalling pathway shown to be involved in early joint formation is the Wnt/beta-catenin pathway. Canonical Wnt signalling is transduced through stabilisation and nuclear accumulation of cytoplasmic beta-catenin where, in conjunction with the lymphoid enhancer-binding factor/transcription factor family binding proteins, it acts as a transcriptional activator [21, 23, 59]. Guo et al. [21] found that several WNT genes, including WNT4, WNT14 (now WNT9A), and WNT16, were expressed in overlapping and complementary patterns in the developing synovial joints, where beta-catenin protein levels and transcription activity were up-regulated. Removal of beta-catenin early in mesenchymal progenitor cells promoted chondrocyte differentiation and blocked the activity of WNT14 in joint formation. Moreover, genetic removal of beta-catenin in chondrocytes led to bone fusion. Thus, according to Guo et al. [21] these results demonstrate that the $\mathrm{Wnt} /$ beta-catenin signalling pathway is necessary and sufficient to induce early steps of synovial joint formation by maintaining joint cell fate and preventing their differentiation to chondrocytes. Similarly, Tamamura et al. [63] established that Wnt/ /beta-catenin signalling regulates chondrocyte phenotype maturation and function in a developmentally regulated manner, and regulated action by this pathway is critical for growth plate organisation, cartilage boundary definition, and endochondral ossification. Day et al. [11] demonstrated also that beta-catenin is essential in determining whether mesenchymal progenitors will become osteoblasts or chondrocytes regardless of regional locations or ossification mechanisms. Controlling Wnt/beta-catenin signalling is a common molecular mechanism underlying chondrocyte and osteoblast differentiation and specification of intramembranous and endochondral ossification. Deficiency in Wnt/beta-catenin signalling causes severe reductions in superficial zone (the outermost layer of articular cartilage) and lubricin gene expression in developing long-bone joint [33]. The Wnt/beta-catenin pathway is initiated by the WNT9A, WNT4, WNT16, and possibly additional WNT ligands [21, 23, 59]. It results in the activation of the genes belonging to the transforming growth factor-beta (TGF-beta) 
superfamily i.e. the growth and differentiation factor 5 (GDF5) and other related bone morphogenetic proteins (BMPs), which contribute together to the specification of synovial joints [62].

Another factor involved in joint development is TGF-beta signalling, which acts upstream of canonical Wnt/beta-catenin signalling $[55,56]$. In mice lacking the TGF-beta type II receptor gene the loss of TGF-beta responsiveness resulted in the absence of interphalangeal joints. TGF-beta receptor II signalling regulates Wnt9a and Gdf5 - joint morphogenic gene expressions [58].

BMPs and GDFs have also a fundamental role during skeletogenesis, including joint formation $[13,16]$. Four members of the BMP/GDF families, BMP-2, and particularly BMP-4, GDF5 and GDF6, are expressed during joint development $[24,61,66]$. GDF5 transcripts are expressed at the sites where joints will later form between skeletal elements. Involvement of GDF5 in joint development was suggested by observation that null mutations in Gdf5 in mice disrupt the formation of more than $30 \%$ of the synovial joints in the limb, leading to complete or partial fusions between particular skeletal elements [61, 62]. Further studies established that GDF5 is expressed not only in the joint interzone, but also in condensing mesenchyme $[3,40]$. Both chondrogenic structures such as articular cartilage and non-chondrogenic structures such as intra-joint ligaments, synovial lining and synovium all derive from the initial population of GDF5-expressing interzone cells $[32,33,45,53]$. Additionally, the BMP activity is controlled by noggin, its antagonist expressed in condensing mesenchymal chondroprogenitor cells and immature chondrocytes. Noggin expression is essential for proper skeletal development since excess BMP activity in the noggin null mutant resulted in excess cartilage and failure to initiate joint formation [9]. At least 2 human syndromes caused by mutations in noggin i.e. proximal symphalangism (SYM1) and multiple synostoses syndrome (SYNS1) characterised by multiple synostoses were identified [20]. As already mentioned a central role in initiating synovial joint formation plays WNT14. It is expressed in joint forming regions (interzones) prior to the segmentation of the cartilage elements and also in the non-chondrogenic mesenchyme but is excluded from the perichondrium [23]. WNT14 expression seems to be sufficient to direct joint development as judged by its ability to induce and/or maintain a panel of gene markers indicative of joint development, including the autotaxin (enzyme important for generating the lipid signalling molecule lysophosphatidic acid), chordin (polypeptide that functions as a BMP antagonist in the developing embryo) or CD44, principal cell surface receptor for hyaluronic acid (HA) [23]. Thus, the joint interzone appears to be an essential regulator of skeletal development, controlling both chondrocytes proliferation and the patterning of chondrocyte differentiation serving as signalling centre $[3,23]$. Moreover, the cells of the interzone are connected by numerous gap junctions formed by junctional protein connexin 32 and 43 , what suggest possibility of information exchange [3].

Indian hedgehog is widely recognised as a critical regulator of long bone development and growth [65] as a target of the bone morphogenetic signalling pathway [54]. Moreover, it also regulates synovial joint formation [60]. In its absence, distribution and function of GDF5 expressing interzone-associated cells are abnormal, but their patterning at prospective joint sites still occurs. The joint-forming functions of these cells appear to normally involve a previously unsuspected asymmetric distribution along the ventral-to-dorsal plane of the developing joint [36]. Lizarraga et al. [38] presented evidence implicating that homeobox containing gene cux 1 , participates in the regulation of the onset of joint formation in the developing chick limb. It is highly expressed at all of the discrete sites of incipient joint formation in the developing limb concomitant with conversion of differentiated chondrocytes into non-chondrogenic cells of the interzone. CUX1 downregulates type II collagen and aggrecan expression in limb chondrocytes but does not induce the expression of either WNT14 or GDF5, signalling molecules that have been implicated in regulating joint formation [23]. This suggests the possibility that cux 1 may be a downstream target gene of these signalling molecules [38].

\section{JOINT CAVITATION}

Process of joint cavitation involves several phenomena. Physiological cell death and degeneration in the interdigital mesenchyme of the hind foot of the rat foetus was observed [6]. Similarly, Mitrovic $[41,42]$ observed in the interzone an early wave of cell necrosis occurring in chicken and rat embryos immediately after it differentiation and suggested that necrotic changes prevented chondrification of 
interzone by clearing up the cells with chondroblastic potentialities. In another study, however, cell death was not observed during cavitation of the developing joints in rat or rabbit knee [28, 31]. Macrophages were present prior to cavitation in the periphery of joint interzones but not at the presumptive joint line in the central region of interzone [14]. Moreover, Edwards et al. [15] did not find evidence that the loss of tissue strength at the joint line which allows synovial joint cavity formation relates to high local levels of metalloproteases.

HA acid may be an important factor in joint cavitation. Activity of uridine diphosphoglucose dehydrogenase, an enzyme necessary for synthesis of UDP glucuronate, a component for HA production was increased in a narrow band of cells at the presumptive joint line prior to cavitation. These findings suggest that joint cavitation is dependent on the behaviour of fibroblastic cells and/or adjacent chondrocytes, rather than macrophages. Moreover, hyaluronan and hyaluronan synthases were present in the interzone before and at the time of cavitation [39]. Thus, joint cavitation could be facilitated by a rise in local hyaluronan concentration in an area of tissue where cohesion is dependent on the interaction between cellular CD44 and extracellular hyaluronan $[4,14$, 49 , 51]. The interaction between HA and CD44 can induce both cell adhesion and cell separation, depending on the concentration of HA surrounding a cell population via receptor saturation, with increasing HA concentrations leading to cell separation [64]. Thus, during limb development, the expression of CD44 in the interzone and at the developing articular surfaces, and the increased HA synthesis associated with cavitation can facilitate tissue separation and create a functional joint cavity $[3,14,49]$. In animals deficient in HA synthase-2, the formation of synovial joint cavities is defective.

Normal joint development requires also proper heparan sulfate production. In mouse embryo mutants with inadequate heparan sulfate synthesis caused by lack of Ext1 (an endoplasmic reticulum-resident type II transmembrane glycosyltransferase involved in the heparan sulfate biosynthesis) the proximal limb joints contained an uneven articulating superficial zone that expressed very low lubricin levels. Digit joints were even more affected, lacked a distinct mesenchymal interzone and were often fused [43].

Important influence on synovial joint formation exerts also developing musculature. Pitsillides [50] and Kahn et al. [30] demonstrated, that the contracting musculature is fundamental in maintaining joint progenitors committed to their fate, and is indispensable for correct joint cavitation and morphogenesis. Molecular mechanism for this regulation is supplied by the contraction-dependent activation of beta-catenin, a key modulator of joint formation.

Specificity of various joints formation seems to depend on an additional, local expression of factors directing joint development. Mice with disrupted paralogous HOX genes (a group of related genes that control the body plan of the embryo along the anterior-posterior axis) had the radius and ulna nearly completely absent [10]. Further studies demonstrated that HOX11 genes regulate also wrist and ankle joint organisation and are essential for establishing carpal and tarsal element boundary and maintaining their articulating surface tissue. It also seems possible that HOX11 genes could favour formation of a thick lubricin-expressing superficial zone around carpal and tarsal elements via direct or indirect stimulation of Wnt/beta-catenin signalling and WNT9A and WNT4 expression and action [34, 35]. The knee is enriched for the hindlimb patterning genes HOXC9, HOXC1O, and several others genes involved in TGF-beta/BMP and Wnt signalling. Thus, it seems that TGF-beta signalling directs knee morphogenesis, and is necessary for meniscus development. Development of elbow may be influenced by genes involved in muscle specification and differentiation since their expression is highly enriched in tissues surrounding the elbow [47].

\section{FORMATION OF ARTICULAR CARTILAGE}

According to the traditional views articular cartilage represents part of embryonic epiphyses that are not replaced by bone through endochondral ossification $[18,26]$. This view was challenged by several authors who assumed that articular cartilage is formed by the cells lying at the periphery of the cavitated joint and derived from the cells of interzone $[4,8,28]$. The authors of these publications showed that the interzone is ultrastructurally distinguishable into 2 outer layers contiguous to the epiphyseal ends and a thin central intermediate zone. Interzone cells from the outer layers were found to differentiate into chondrocytes early in embryogenesis and become incorporated into the epiphyses, thus contributing to initial lengthening of the anlagen. Cells deriving from the intermediate zone were instead found to remain located in the joint proper and become arti- 
cular cartilage. Hyde et al. [27] used matrilin-1 (one of the cartilage matrix proteins) which is expressed by epiphyseal chondrocytes but not by articular chondrocytes $[5,44]$ to demonstrate that articular chondrocytes are derived from cells that have never expressed matrilin-1. The remainder of the chondrocytes in the cartilage anlagen express matrilin-1. A band of chondrocytes adjacent to the developing interzone in the developing mouse knee joint became apparent because these chondrocytes did not turn on expression of matrilin-1 in contrast to the other chondrocytes of the anlagen. The chondrocytes of the presumptive articular surface therefore appear to arise directly from a subpopulation of early chondrocytes that do not activate matrilin-1 expression rather than by redifferentiation from the perichondrium-like layer cells of the interzone. In further studies Pacifici et al. [46] and Koyama et al. [33] unexpectedly found that peri-joint mesenchymal cells take part in interzone formation, and that these interzone cells expressing Gdf5 constitute a distinct cohort of progenitor cells. These cells also expressed genes such as Wnt9a and for collagen IIA, remained predominant in the joint-forming sites over time, gave rise to articular cartilage, synovial lining and other joint tissues, but contributed little if any to underlying growth plate cartilage and shaft. In mutants deficient in $\mathrm{Wnt} /$ /beta-catenin signalling joints did form, but displayed a defective flat cell layer normally abutting the synovial cavity and expressed markedly reduced levels of lubricin. Additionally acquisition of a permanent articular chondrocyte phenotype was aided and perhaps dictated by transcription factor ERG belonging to the ETS family of transcription factors (reviewed in [57]). Thus, according to Pacifici et al. [46] and Koyama et al. [43] articular chondrocytes have a distinct embryonic origin as compared to the chondrocytes that form cartilage anlagen for the developing bones. Anlage chondrocytes have a transient character, proliferate, undergo hypertrophy and apoptosis prior to endochondral bone formation and finally disappear after cessation of growth plate function. On the other hand, articular chondrocytes have stable and permanent phenotype and function throughout life. Nevertheless, this phenotype may be disturbed, since in osteoarthritic articular cartilage chondrocytes expression of early (proliferating cell nuclear antigen, syndecan-3) and late (annexin VI, alkaline phosphatase) differentiation markers was activated, suggesting that they acquire features of transient phenotype [48]. Moreover, BMP receptor signalling is required not only for early development and creation of multiple joint tissues, but also for ongoing maintenance of articular cartilage after birth. Genetic variation in the strength of BMP receptor signalling may be an important risk factor in human osteoarthritis, and treatments that mimic or augment BMP receptor signalling should be investigated as a possible therapeutic strategy for maintaining the health of joint linings [53].

\section{ACKNOWLEDGEMENTS}

Publication was funded by the National Science Centre (Poland) on the basis of decision number: DEC-2012/05/B/NZ4/02646.

\section{REFERENCES}

1. Akiyama H, Chaboissier MC, Martin JF, Schedl A, de Crombrugghe $B$ (2002) The transcription factor Sox 9 has essential roles in successive steps of the chondrocyte differentiation pathway and is required for expression of Sox 5 and Sox6. Genes Dev, 16: 2813-2828.

2. Andersen $H$, Bro-Rasmussen $F(1961)$ Histochemical studies on the histogenesis of the joints in human fetuses. Am J Anat, 108: 111-122.

3. Archer CW, Dowthwaite GP, Francis-West P (2003) Development of synovial joints. Birth Defects Res (Part C), 69: 144-155.

4. Archer CW, Morrison H, Pitsillides AA (1994) Cellular aspects of the development of diarthrodial joints and articular cartilage. J Anat, 184: 447-456.

5. Aszodi A, Bateman JF, Hirsch E, Baranyi M, Hunziker EB, Hauser N, Bosze Z, Fassler R (1999) Normal skeletal development of mice lacking matrilin 1: redundant function of matrilins in cartilage? Mol Cell Biol, 19: 7841-7845.

6. Ballard KJ, Holt SJ (1968) Cytological and cytochemical studies on cell death and digestion in the fetal rat foot: the role of macrophages and hydrolytic enzymes. J Cell Sci, 3: 245-262.

7. Bi W, Deng JM, Zhang Z, Behringer RR, de Crombrugghe B (1999) Sox9 is required for cartilage formation. Nat Genet, 22: 85-89.

8. Bland YA, Ashhurst DE (1996) Development and ageing of the articular cartilage of the rabbit knee joint: distribution of fibrillar collagens. Anat Embryol, 194: 607-619.

9. Brunet $\sqcup$, McMahon JA, McMahon AP, Harland RM (1998) Noggin, cartilage morphogenesis, and joint formation in the mammalian skeleton. Science, 280: 1455-1457.

10. Davis AP, DP Witte, Hsieh-Li HM, Potter SS, Capecchi MR (1995) Absence of radius and ulna in mice lacking hoxa-11 and hoxd-11. Nature, 375: 791-795.

11. Day TF, Guo X, Garrett-Beal L, Yang Y (2005) Wnt/beta-catenin signaling in mesenchymal progenitors controls osteoblast and chondrocyte differentiation during vertebrate skeletogenesis. Dev Cell, 8: 739-750.

12. Dy $P$, Smits $P$, Silvester $A$, Penzo-Méndez $A$, Dumitriu $B$, Han Y, de la Motte CA, Kingsley DM, Lefebvre V (2010) Synovial joint morphogenesis requires the chondrogenic action of Sox 5 and Sox 6 ingrowth plate and articular cartilage. Dev Biol, 341: 346-359.

13. Edwards JCW, Francis-West PH (2001) Bone morphogenetic proteins in the development and healing of synovial joints. Semin Arthritis Rheum, 31: 33-42. 
14. Edwards JCW, Wilkinson LS, Jones HM, Soothill P, Henderson KJ, Worrall JG, Pitsillides AA (1994) The formation of human synovial joint cavities: a possible role for hyaluronan and CD44 in altered interzone cohesion. J Anat, 185: 355-367.

15. Edwards JCW, Wilkinson LS, Soothill P, Hembry RM, Murphy G, Reynolds JJ (1996) Matrix metalloproteinases in the formation of human synovial joint cavities. J Anat, 188: 355-360.

16. Francis-West PH, Abdelfattah $A$, Chen $P$, Allen $C$, Parish J, Ladher R, Allen S, MacPherson S, Luyten FP, Archer CW (1999) Mechanisms of GDF-5 action during skeletal development. Development, 126: 1305-1315.

17. Francis-West PH, Parish J, Lee K, Archer CW (1999) BMP/ /GDF-signaling interactions during synovial joint development. Cell Tissue Res, 296: 111-119.

18. Goldring MB (2012) Chondrogenesis, chondrocyte differentiation, and articular cartilage metabolism in health and osteoarthritis. Ther Adv Musculoskel Dis, 4: 269-285.

19. Goldring MB, Tsuchimochi K, ljiri K (2006) The control of chondrogenesis. J Cell Biochem, 97: 33-44.

20. Gong Y, Krakow D, Marcelino J, Wilkin D, Chitayad D, Babul-Hyriji R, Hudgins L, Cremers CW, Cremers FP, Brunner HG, Reinker K, Rimoin DL, Cohn DH, Goodman FR, Reardon W, Patton M, Francomano CA, Warman ML (1999) Heterozygous mutations in the gene encoding noggin affect human joint morphogenesis. Nat Genet, 21: 302-304.

21. Guo X, Day TF, Jiang X, Garrett-Beal L, Topol L, Yang Y (2004) $\mathrm{Wnt} /$ beta-catenin signaling is sufficient and necessary for synovial joint formation. Genes Dev, 18: 2404-2417.

22. Han Y, Lefebvre V (2008) L-Sox5/Sox6 drive expression of the aggrecan gene in cartilage by securing binding of Sox 9 to a far-upstreamenhancer. Mol Cell Biol, 28: 4999-5013.

23. Hartmann C, Tabin CJ (2001) Wnt-14 plays a pivotal role in inducing synovial joint formation in the developing appendicular skeleton. Cell, 104: 341-351.

24. Hogan BL (1996) Bone morphogenetic proteins: multifunctional regulators of vertebrate development. Genes Dev, 10: 1580-1594.

25. Holder N (1977) An experimental investigation into the early development of the chick elbow joint. J Embryol Exp Morphol, 39: 115-127.

26. Hunziker EB (1988) Growth plate structure and function. Pathol Immunopathol Res, 7: 9-13.

27. Hyde G, Dover S, Aszodi A, Wallis GA, Boot-Handford RP (2007) Lineage tracingusing matrilin-1 gene expression reveals that articular chondrocytes exist as the joint interzone forms. Dev Biol, 304: 825-833.

28. Ito MM, Kido MY (2000) Morphological and biochemical re-evaluation of the process of cavitation in the rat knee joint: cellular and cell strata alterations in the interzone. J Anat, 197: 659-679.

29. Iwamoto M, Tamamura Y, Koyama ., Komori T, Takeshita N, Williams JA, Nakamura T, Enomoto-Iwamoto M, Pacifici M (2007) Transcription factor ERG and joint and articular cartilage formation during mouse limb and spine skeletogenesis. Dev Biol, 305: 40-51.

30. Kahn J, Shwartz Y, Blitz E, Krief S, Sharir A, Breitel DA, Rattenbach R, Relaix F, Maire P, Roundtree RB, Kingsley DM, Zelzer $E$ (2009) Muscle contraction is necessary to maintain joint progenitor cell fate. Dev Cell, 16: 734-743.
31. Kavanagh E, Abiri M, Bland YS, Ashhurst DE (2002) Division and death of cells in developing synovial joints and long bones. Cell Biol Int, 26: 679-688.

32. Koyama E, Ochiai T, Rountree RB, Kingsley DM, Enomoto-Iwamoto M, Iwamoto M, Pacifici M (2007) Synovial joint formation during mouse limb skeletogenesis. Roles of Indian hedgehog signaling. Ann NY Acad Sci, 1116: 100-112.

33. Koyama E, Shibukawa $Y$, Nagayama M, Sugito H, Young B, Yuasa $T$, Okabe T, Ochiai T, Kamiya N, Rountree RB, Kingsley DM, Iwamoto M, Enomoto-Iwamoto M, Pacifici M (2008) A distinct cohort of progenitor cells participates in synovial joint and articular cartilage formation during mouse limb skeletogenesis. Dev Biol, 316: 62-73.

34. Koyama E, Yasuda T, Minugh-Purvis N, Kinumatsu T, Yallowitz AR, Wellik DM, Pacifici M (2010) Hox11 genes establish synovial joint organization and phylogenetic characteristics in developing mouse zeugopod skeletal elements. Development, 137: 3795-3800.

35. Koyama E, Yasuda T, Wellik DM, Pacifici M (2010) Hox11 paralogous genes are required for formation of wrist and ankle joints and articular surface organization. Ann NY Acad Sci, 1192: 307-316.

36. Koyama E, Young B, Nagayama M, Shibukawa Y, Enomoto-Iwamoto M, Iwamoto M, Maeda Y, Lanske B, Song B, Serra R, Pacifici M (2007) Conditional Kif3a ablation causes abnormal hedgehog signaling topography, growth plate dysfunction, and excessive bone and cartilage formation during mouse skeletogenesis. Development, 134: 2159-2169.

37. Lefebvre V, Smits P (2005) Transcriptional control of chondrocyte fate and differentiation. Birth Defects Res (Part C), 75: $200-212$.

38. Lizarraga G, Lichtler A, Upholt WB, Kosher RA (2002) Studies on the role of Cux1 in regulation of the onset of joint formation in the developing limb. Dev Biol, 243: 44-54.

39. Matsumoto $K$, Yingcui $L$, Jakuba $C$, Sugiyama $Y$, Sayo $T$, Okuno M, Dealy CN, Toole BP, Takeda J, Yamaguchi Y, Kosher RA (2009) Conditional inactivation of Has2 reveals a crucial role for hyaluronan in skeletal growth, patterning, chondrocyte maturation and joint formation in the developing limb. Development, 136: 2825-2835.

40. Merino R, Macias D, Ganan $Y$, Economides AN, Wang $X$, Wu Q, Stahl N, Sampath KT, Varona P, Hurle JM (1999). Expression and function of Gdf 5 during digit skeletogenesis in the embryonic chick leg bud. Dev Biol, 206: 33-45.

41. Mitrovic D (1977). Development of the metatarsophalangeal joint of the chick embryo: Morphological, ultrastructural and histochemical studies. Am J Anat, 150: 333-348.

42. Mitrovic D (1978) Development of the diarthrodial joints in the rat embryo. Am Anat, 151: 475-486.

43. Mundy C, Yasuda T, Kinumatsu T, Yamaguchi Y, Iwamoto M, Enomoto-Iwamoto M, Koyama E, Pacifici M (2011) Synovial joint formation requires local Ext1 expression and heparan sulfate production in developing mouse embryo limbs and spine. Dev Biol, 351: 70-81.

44. Murphy JM, Heinegard D, Mclntosh A, Sterchi D, Barry FP (1999) Distribution of cartilage molecules in the developing mouse joint. Mat Biol,18: 487-497.

45. Pacifici M, Koyama E, Iwamoto M (2005) Mechanisms of synovial joint and articular cartilage formation: recent advances, but many lingering mysteries. Birth Defects Res (Part C), 75: 237-248. 
46. Pacifici M, Koyama E, Shibukawa Y, Wu C, Takamura Y, Enomoto-Iwamoto M, Iwamoto M (2006) Cellular and molecular mechanisms of synovial joint and articular cartilage formation. Ann NY Acad Sci, 1068: 74-86.

47. Pazin DE, Gamer LW, Cox KA, Rosen V (2012) Molecular profiling of synovial joints: use of microarray analysis to identify factors that direct the development of the knee and elbow. Dev Dyn, 241: 1816-1826.

48. Pfander D, Swoboda B, Kirsch T (2001) Expression of early and late differentiation markers (proliferating cell nuclear antigen, syndecan-3, annexin VI and alkaline phosphatase) by human ostearthritic chondrocytes. Am J Path, 159: 1777-1783.

49. Pitsillides AA (2003) Identifying and characterizing the joint cavity-forming cell. Cell Biochem Funct, 21: 235-240.

50. Pitsillides AA (2006) Early effects of embryonic movement: "a shot out of the dark". J Anat, 208: 417-431.

51. Pitsillides AA, Archer CW, Prehm P, Bavliss MT, Edwaeds JC (1995) Alterations in hyaluronan synthesis during developing joint cavitation. J Histochem Cytochem, 43: 263-273.

52. Pitsillides AA, Ashhurst DE (2008) A critical evaluation of specific aspects of joint development. Dev Dyn, 237: 2284-2294.

53. Rountree RB, Schoor M, Chen $H$, Marks ME, Harley $V$, Mishina Y, Kingsley DM (2004) BMP receptor signaling is required for postnatal maintenance of articular cartilage. PLoS Biol, 2: 1815-1827.

54. Seki K, Hata A (2004) Indian Hedgehog gene is a target of the bone morphogenetic protein signaling pathway. J Biol Chem, 279: 18544-18549.

55. Seo HS, Serra R (2007) Deletion of Tgfbr2 in Prx1-cre expressing mesenchyme results in defects in development of the long bones and joints. Dev Biol, 310: 304-316.

56. Serra R, Chan C (2003) TGF-beta signaling in human skeletal and patterning disorders. Birth Defects Res (Part C), 69: 333-351.
57. Sharrocks AD (2001) The ETS-domain transcription factor family. Nat Rev Mol Cell Biol, 2: 827-837.

58. Spagnoli A, O'Rear L, Chandler RL, Granero-Molto F, Mortlock DP, Gorska AE, Weis JA, Longobardi L, Chytil A, Shimer K, Moses HL (2007) TGF-beta signaling is essential for joint morphogenesis. J Cell Biol, 177: 1105-1117.

59. Später D, Hill TP, Gruber M, Hartmann C (2006) Role of canonical Wnt-signaling in joint formation. Eur Cell Mater, 12: 71-80.

60. St-Jacques B, Hammerschmidt M,. Mcmahon AP (1999) Indian hedgehog signaling regulates proliferation and differentiation of chondrocytes and is essential for bone formation. Genes Dev, 13: 2076-2086.

61. Storm EE, Kingsley DM (1996) Joint patterning defects caused by single and double mutations in members of the bone morphogenetic protein (BMP) family. Development, 122: 3969-3979.

62. Storm EE, Kingsley DM (1999) GDF5 coordinates bone and joint formation during digit development. Dev Biol, 209: 11-27.

63. Tamamura $Y$, Otani $T$, Kanatani N, Koyama E, Kitagaki J, Komori T, Yamada Y, Costantini F, Wakisaka S, Pacifici M, Iwamoto M, Enomoto-Iwamoto M (2005). Developmental regulation of $\mathrm{Wnt} /$ beta-catenin signals is required for growth plate assembly, cartilage integrity, and endochondral ossification. J Biol Chem, 280: 19185-19195.

64. Toole BP (1991) Glycosaminoglycans in morphogenesis. In: Hay ED ed. Cell biology of the extracellular matrix. Plenum Press, New York, pp. 259-294.

65. Vortkamp A, Lee K, Lanske B, Segre GV, Kronenberg HM, Tabin CJ (1996) Regulation of rate of cartilage differentiation by Indian hedgehog and PTH-related protein. Science, 273: 613-622.

66. Zou H, Wieser R, Massagué J, Niswander L (1997) Distinct roles of type I bone morphogenetic protein receptors in the formation and differentiation of cartilage. Genes Dev, 11: 2191-2203. 